\title{
Sensitivity to Interaural Time Differences in the Inferior Colliculus with Bilateral Cochlear Implants
}

\author{
Zachary M. Smith ${ }^{1,2}$ and Bertrand Delgutte ${ }^{1,2,3}$ \\ ${ }^{1}$ Eaton-Peabody Laboratory, Massachusetts Eye and Ear Infirmary, Boston, Massachusetts 02114, ${ }^{2}$ Speech and Hearing Bioscience and Technology \\ Program, Harvard-Massachusetts Institute of Technology (MIT) Division of Health Sciences and Technology, Cambridge, Massachusetts 02139, and \\ ${ }^{3}$ Research Laboratory of Electronics, MIT, Cambridge, Massachusetts 02139
}

\begin{abstract}
Bilateral cochlear implantation attempts to increase performance over a monaural prosthesis by harnessing the binaural processing of the auditory system. Although many bilaterally implanted human subjects discriminate interaural time differences (ITDs), a major cue for sound localization and signal detection in noise, their performance is typically poorer than that of normal-hearing listeners. We developed an animal model of bilateral cochlear implantation to study neural ITD sensitivity for trains of electric current pulses delivered via bilaterally implanted intracochlear electrodes. We found that a majority of single units in the inferior colliculus of acutely deafened, anesthetized cats are sensitive to ITD and that electric ITD tuning is as sharp as found for acoustic stimulation with broadband noise in normal-hearing animals. However, the sharpness and shape of ITD tuning often depended strongly on stimulus intensity; some neurons had dynamic ranges of ITD sensitivity as low as $1 \mathrm{~dB}$. We also found that neural ITD sensitivity was best at pulse rates below $100 \mathrm{~Hz}$ and decreased with increasing pulse rate. This rate limitation parallels behavioral ITD discrimination in bilaterally implanted individuals. The sharp neural ITD sensitivity found with electric stimulation at the appropriate intensity is encouraging for the prospect of restoring the functional benefits of binaural hearing in bilaterally implanted human subjects and suggests that neural plasticity resulting from previous deafness and deprivation of binaural experience may play a role in the poor ITD discrimination with current bilateral implants.
\end{abstract}

Key words: binaural hearing; electric stimulation; neural prosthesis; cochlear implant; inferior colliculus; ITD

\section{Introduction}

Tens of thousands of people worldwide have been treated for deafness by cochlear implantation in a single ear. These cochlear implant users often have remarkably good speech reception in quiet, but their speech understanding drops precipitously in the presence of competing sounds common in the everyday acoustic world (Hochberg et al., 1992; Fu et al., 1998). Binaural hearing normally provides many functional advantages over monaural hearing, including improved speech reception in noise and more accurate sound localization in lateral angle (Durlach and Colburn, 1978; Blauert, 1997). In recent years, cochlear implant candidates have increasingly been implanted in both ears in the hope of restoring the functional benefits of binaural hearing. A key issue facing the design of bilateral cochlear implant systems is how acoustic information should be encoded into electric stimuli so that patients obtain a maximal benefit. To identify fundamental physiological limitations on the effective delivery of binaural information, we have developed an animal model of bilateral cochlear implants for studying responses of binaural neurons in

Received Jan. 5, 2007; revised April 19, 2007; accepted May 4, 2007.

This work was supported by National Institutes of Health (NIH) Grants R01 DC005775 and P30 DC005209. Z.M.S was partially supported by NIH Grant T32 DC00038. We thank Connie Miller for surgical assistance, Ken Hancock for computer support, and Steve Colburn and Don Eddington for valuable comments on this manuscript.

Correspondence should be addressed to Dr. Zachary M. Smith, Department of Biomedical Engineering, Johns Hopkins University, 505 Traylor Building, 720 Rutland Avenue, Baltimore, MD 21205. E-mail:zsmith@alum.mit.edu. DOI:10.1523/JNEUROSCI.0052-07.2007

Copyright $\odot 2007$ Society for Neuroscience $\quad$ 0270-6474/07/276740-11\$15.00/0 the auditory midbrain. The primary focus is on the coding of interaural time difference (ITD) because ITD is the dominant cue used for azimuthal localization of sounds containing lowfrequency energy such as speech (Wightman and Kistler, 1992; Macpherson and Middlebrooks, 2002), and because binaural advantages in speech intelligibility in noise depend primarily on the target and interferer having distinct ITDs (Zurek, 1993).

Psychophysical studies in bilaterally implanted subjects show significant improvements in both sound localization and speech intelligibility in spatially separated noise with bilateral over monolateral stimulation (Muller et al., 2002; Schon et al., 2002; van Hoesel et al., 2002; van Hoesel and Tyler, 2003; Litovsky et al., 2006). Direct tests of sensitivity to interaural level differences (ILDs) show relatively good discrimination thresholds that can be as low as the smallest ILD $(\sim 0.1-0.2 \mathrm{~dB})$ allowed by clinical implant systems (Lawson et al., 1998; Long et al., 2003; van Hoesel and Tyler, 2003). In contrast, ITD discrimination thresholds are highly variable, ranging from $50 \mu \mathrm{s}$ (rare) to several milliseconds depending on the subject, pulse rate, and electrode pair tested (Lawson et al., 1998; van Hoesel and Tyler, 2003; Wilson et al., 2003; van Hoesel, 2007). In comparison, discrimination of ITD for acoustic stimuli in normal-hearing subjects is better, more consistent across subjects, and less dependent on stimulus parameters, with just noticeable differences (JNDs) of 10-20 $\mu \mathrm{s}$ for clicks (Klumpp and Eady, 1956; Yost et al., 1971). Thus, ITD coding is where there is the most room for improvement in current bilateral implants. 
In the present study, we investigated the ITD sensitivity of inferior colliculus (IC) neurons to electric pulse trains. Stimuli were delivered via bilaterally implanted electrodes in the cochleae of acutely deafened, anesthetized cats. ITD tuning with electric stimulation is compared with published data for acoustic stimulation in normal-hearing animals. Neural ITD sensitivity is also quantified using detection theoretic metrics and compared with behavioral ITD thresholds in human subjects with bilateral cochlear implants. Preliminary reports of this work have been presented (Smith and Delgutte, 2003a,b, 2005a,b).

\section{Materials and Methods}

Subjects and deafening. All surgical and experimental procedures followed the regulations set by the National Institutes of Health and were approved by the internal animal care and use committees of both the Massachusetts Eye and Ear Infirmary and the Massachusetts Institute of Technology. Healthy adult cats of either sex were deafened by coadministration of kanamycin $(300 \mathrm{mg} / \mathrm{kg}$, s.c. $)$ and ethacrynic acid $(25 \mathrm{mg} / \mathrm{kg}$, i.v.) 7-14 d before cochlear implantation and electrophysiological recordings (Xu et al., 1993).

Effectiveness of the deafening protocol was assessed on the day of the experiment by measuring auditory brainstem response (ABR) to acoustic clicks in each ear. The animals were anesthetized and surgically prepared as described below. Calibrated acoustic assemblies comprising an electrodynamic speaker and a probe-tube microphone were inserted into the cut ends of each ear canal to form a closed system. Condensation clicks $(100 \mu \mathrm{s})$ were delivered via these acoustic systems, and ABR thresholds measured in both ears. ABR was measured between vertex and ear bar using a small screw inserted into the skull. In all experiments, no ABR response was seen up to the highest intensity tested (110 dB sound pressure level peak).

Surgery. On the day of the experiment, after induction of anesthesia by Dial in urethane $(75 \mathrm{mg} / \mathrm{kg})$, a tracheal cannula was inserted; skin and muscles overlying the back and top of the skull were reflected. Ear canals were transected for insertion of a calibrated closed acoustic system. Tympanic bullae were opened to allow access to the round window for implanting intracochlear electrodes. Part of the skull overlying the occipital cortex was removed to allow for partial aspiration of cortical tissue and access to the IC. The part of the bony tentorium overlying the IC was drilled for better access to the dorsolateral surface of the IC. Throughout all procedures, animals were given supplementary doses of anesthesia to maintain an areflexic state, and vital signs were monitored.

Cochlear implantation and electrode configurations. Stimulating electrodes were surgically implanted into each cochlea through the exposed round window. The electrodes were either custom-made Pt/Ir ball electrodes $(0.45 \mathrm{~mm}$ diameter) or eight-contact electrode arrays with 0.75 $\mathrm{mm}$ spacing (ring-type contacts with $0.45 \mathrm{~mm}$ diameter; Cochlear Corporation, Englewood, CO). Particular care was taken to achieve the same insertion depth on both sides. In all experiments, we used a wide bipolar configuration ( $\sim 5 \mathrm{~mm}$ between electrodes), with the active electrode inserted $\sim 5 \mathrm{~mm}$ into the scala tympani and the return electrode (either another Pt/Ir ball or the most basal contact of the array) just inside the round window. This electrode configuration is similar to the monopolar configuration commonly used in clinical devices in that it produces a broad pattern of excitation but reduces the stimulus artifact measured at the recording electrodes by decreasing the distance between the active and return electrodes (Litvak et al., 2003). With this type of stimulation, we observed spatial excitation patterns with a single maximum across the tonotopic axis of the IC.

Single-unit recordings and cancellation of stimulus artifact. Single-unit activity in the IC was recorded using either parylene-insulated tungsten stereotrodes ( $\sim 2 \mathrm{M} \Omega$ impedance; Microprobe, Potomac, MD) or 16channel silicon probes (100 or $150 \mu \mathrm{m}$ spacing between contacts, 177 $\mu \mathrm{m}^{2}$ site area; NeuroNexus Technologies, Ann Arbor, MI). Recording electrodes were advanced through the IC along either of two possible trajectories. The "standard" trajectory ran from dorsolateral to ventromedial in the coronal plane tilted $45^{\circ}$ off the sagittal plane so as to record activity from a set of neurons covering a highly reproducible range of characteristic frequencies (CFs) (Merzenich and Reid, 1974; Snyder et al., 1990). The alternate electrode trajectory ran vertically from dorsal to ventral and is referred to as the vertical trajectory. The vertical trajectory was used in an effort to sample more neurons in the lateral part of the IC where the majority of projections from the medial superior olive (MSO) are located (Aitkin and Schuck, 1985; Oliver et al., 2003).

Stimulus artifact (the electric potential seen at the recording electrode directly resulting from current passing between the stimulating electrodes) was typically much larger than the single-unit activity. Recordings were made simultaneously from two neighboring sites, only one of which sampled the activity of the neuron of interest. Because stimulus artifact was present in both channels, it could be minimized by filtering and subtracting the reference channel (which only contains artifact) from the single-unit channel with an adaptive filter. Real-time artifact cancellation was achieved using a least-mean-square adaptive filter (Widrow et al., 1975), implemented in software running on a digital signal processing board (Tucker-Davis Techonologies, Alachua, FL).

After on-line artifact cancellation, spikes from well isolated single units were amplified and detected with a custom-built discriminator. Spike times were measured by a custom-built timer with a resolution of 1 $\mu$ s and stored to disk for additional processing and display.

Stimulus generation and delivery. All stimuli were generated by a pair of 16-bit digital-to-analog converters (NIDAC 6052e; National Instruments, Austin, TX) using a sampling rate of $100 \mathrm{kHz}$. Stimulus levels were set by custom-built attenuators having a resolution of $0.1 \mathrm{~dB}$. Attenuated outputs of the digital-to-analog converters were delivered to the intracochlear electrodes via a pair of custom-built, high-bandwidth $(40 \mathrm{kHz})$, optically isolated constant-current sources. All electric stimuli were made up of $100 \mu$ s biphasic current pulses (cathodic-anodic, $50 \mu$ s per phase). Current levels are expressed in decibels relative to (re.) $1 \mathrm{~mA}$ and reflect the peak amplitude of the current pulses. We use the term "intensity" somewhat freely here to refer to peak current level.

The search stimulus consisted of a sequence of three current pulses with a $100 \mathrm{~ms}$ interval between each pulse. The first two pulses were delivered monaurally to each ear in turn, and the last pulse was binaural. The search stimulus was repeated at a rate of two per second at an intensity well above the threshold of local field potentials. Once a single unit was isolated, its threshold was measured with monaural and binaural pulses. After this preliminary characterization of the unit, ITD sensitivity was studied.

ITD stimuli. Both static and dynamic ITD stimuli were used to assess ITD tuning with pulse trains at 40 pulses per second (pps). For static ITD stimuli, a $300 \mathrm{~ms}$ stimulus containing 12 pulses was repeated 10 times for each ITD with a $200 \mathrm{~ms}$ silent interval between trials. The typical range of ITDs tested with static stimuli was $-1000 \mu$ s to $+1000 \mu$ s in $100 \mu$ s steps. Positive ITDs refer to a stimulus leading in the contralateral ear. Stimuli with dynamic ITDs are commonly used to efficiently study ITD sensitivity in single units (Kuwada et al., 1979). Our dynamic stimuli had an ITD that changed in a triangular pattern with each successive pulse in the train, starting at $-1000 \mu \mathrm{s}$, going up to $+1000 \mu \mathrm{s}$, and then back to $-1000 \mu$ s in $100 \mu$ s steps. Figure $1 B$ (dotted line) shows the evolution of dynamic ITD throughout the stimulus. Each forward and reverse sweep of the dynamic ITD stimulus was $1050 \mathrm{~ms}$ in duration. The stimulus was repeated 20 times without any interruption for a total duration of $21 \mathrm{~s}$ and 40 presentations of each ITD step. A larger range of ITD (e.g., \pm 2000 $\mu \mathrm{s})$ was occasionally used with the static and dynamic ITD stimuli for neurons with relatively broad ITD tuning. ITD tuning was studied at multiple intensities in most neurons with the dynamic stimulus. Because ITD tuning often changed with intensity, a standard intensity, defined as the lowest intensity that elicited a spike rate $>0.5$ spikes/pulse for at least one ITD, was used for making comparisons of ITD tuning across neurons.

In some neurons, the effect of ILD on ITD tuning was also studied. ILD was typically varied over a $\pm 3 \mathrm{~dB}$ range by increasing the current level in one ear while decreasing the current level in the other ear. Current level changes in each ear were of equal magnitude but opposite sign, thereby maintaining a fixed mean binaural level (MBL; defined as the arithmetic mean of the current levels in decibels at both ears). A similar method for 
varying ILD is widely used with acoustic stimulation (Semple and Kitzes, 1987; Irvine and Gago, 1990; Delgutte et al., 1999).

Data analysis. Spike times were processed to compute average firing rates as a function of ITD. For static ITD stimuli, average firing rates were computed by counting the spikes occurring over the $300 \mathrm{~ms}$ duration of the stimulus in each trial. For the dynamic stimulus, spikes occurring during the $25 \mathrm{~ms}$ interval between consecutive pulses were assigned to the ITD of the leading pulse.

A response modulation depth $(\mathrm{MD})$ was defined for each rate-ITD curve:

$$
\mathrm{MD}=\frac{\max (\text { rate })-\min (\text { rate })}{\max (\text { rate })} .
$$

A unit was classified as being ITD sensitive if the MD of its rate-ITD curve for $40 \mathrm{pps}$ pulse trains was at least 0.5 at an intensity that evoked at least 10 spikes/s.

Rate-ITD curves could have a wide variety of shapes, including peak, trough, sigmoidal, biphasic, and multimodal shapes. To characterize these shapes and obtain quantitative metrics of ITD sensitivity, each rate-ITD curve was fit with three different equations, and the best-fitting equation was used to classify the ITD curve into one of five types. Specifically, peak- and trough-shaped ITD curves were those best fit by Gaussian functions of the following form:

$$
\operatorname{Rate}(\mathrm{ITD})=A 2^{-\left(\frac{I T D-I T D_{b e s t}}{H W / 2}\right)^{2}}+B
$$

where $I T D_{\text {best }}$ is the best ITD, $H W$ is the half-width, and $A$ and $B$ are scaling factors. Positive values of $A$ give peak-shaped ITD curves (see Fig. $2 A$ ), whereas negative values give trough-shaped curves (see Fig. $2 B$ ). Monotonic ITD curves (see Fig. $2 C$ ) were those best fit with a sigmoid function of the following form:

$$
\text { Rate }(\text { ITD })=\frac{A}{1+3^{-2\left(\frac{I T D-I T D_{M S}}{H R}\right)}}+B,
$$

where $I T D_{M S}$ is the ITD of maximum slope, $H R$ is the half-rise, and $A$ and $B$ are, again, scaling factors. Finally, ITD responses with biphasic shapes (see Fig. 2D) were best fit with a difference of two Gaussians (DOG):

$$
\operatorname{Rate}(\mathrm{ITD})=A\left[e^{-\left(\frac{I T D-I T D_{M S}-D}{D}\right)^{2}}-e^{-\left(\frac{I T D-I T D_{M S}+D}{D}\right)^{2}}\right]+B,
$$

where $I T D_{M S}$ is, again, the ITD of maximum slope and $D$ characterizes the steepness of the rise from minimum to maximum. ITD curves that were poorly fit $\left(r^{2}<0.75\right)$ by all three functions (Gaussian, sigmoid, and DOG) were classified as multipeaked because they usually showed several maxima.

Neural discrimination. We used a metric from signal detection theory to estimate ITD discrimination thresholds of single neurons in a way that can be compared with psychophysical thresholds (Shackleton et al., 2003). Specifically, our metric of neural discrimination was a slightly modified version of standard separation (Sakitt, 1973), or D, and was defined as follows:

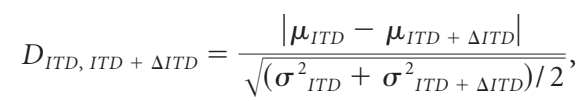

where $\mu_{\mathrm{ITD}}$ and $\mu_{\mathrm{ITD}+\Delta \mathrm{ITD}}$ are the means of the spike counts and $\sigma_{\mathrm{ITD}}$ and $\sigma_{\text {ITD }+\Delta \text { ITD }}$ are their respective SDs. This metric expresses the difference in spike counts elicited by stimuli at two different ITDs in units of their combined SD. We replaced the geometric mean of variances in the original definition of $D$ with the arithmetic mean to avoid problems when the spike-count variance is 0 for one of the ITDs. Standard separation is analogous to $d^{\prime}$, which is often used to quantify discrimination in psychophysical studies (Green and Swets, 1966). The JND in ITD was defined as the $\triangle$ ITD needed for the standard separation to reach a value of 1 . This criterion corresponds to $76 \%$ correct in a two-interval, twoalternative forced-choice discrimination task.

\section{Results}

Results are based on responses of 140 IC neurons to bilateral electric stimulation of the cochlea in 21 deafened cats. The responses to electric pulses had short latencies (mean latencies typically 5-10 ms), consistent with a previous study of the IC in acutely deafened animals (Shepherd et al., 1999). Thus, it is likely that most of our recordings were from the central nucleus of the IC (ICC). We first describe the basic responses of IC neurons as a function of ITD for 40 pps pulse-train stimuli and then look at the influence of overall stimulus intensity, ILD, and stimulus pulse rate on ITD tuning. We then estimate neural ITD discrimination thresholds for single neurons and compare them with behavioral thresholds from human bilateral cochlear implant subjects (van Hoesel and Tyler, 2003; Poon, 2006).

\section{Comparison of responses to static and dynamic ITD stimuli}

Of the 140 neurons studied, 121 (86\%) were considered ITD sensitive $(M D, \geq 0.5)$ for a 40 pps pulse train with either a static or a dynamic ITD. The dynamic stimulus allowed for rapid characterization of the ITD tuning. Temporal discharge patterns from one neuron in response to both static and dynamic ITD stimuli are shown in Figure 1. The dot raster display for the static ITD stimulus (Fig. $1 A$ ) shows that the neuron responds over a limited range of ITD $(-100$ to $+200 \mu \mathrm{s})$ with spikes tightly time-locked to the individual stimulus pulses. The dot raster for the dynamic stimulus (Fig. $1 B$ ) shows that spikes tend to occur when the instantaneous value of ITD is between -100 and $+200 \mu \mathrm{s}$, consistent with responses to the static stimulus. The shapes of the rate-ITD curves derived from the responses to the two stimuli are very similar, suggesting that dynamically varying ITD has little effect on ITD tuning for this unit. However, the peak firing rate for the dynamic stimulus is somewhat larger.

Figure $1 C$ compares rate-ITD curves measured with static and dynamic-ITD stimuli for eight neurons. For most neurons, the rate-ITD curves have similar shapes for both stimuli. However, there are obvious differences in overall spike rate for some of the neurons, and in some cases (Fig. 1C, top left panel), ITD tuning is broader for the static stimulus. Figure $1 D$ shows the best ITD $\left(\mathrm{ITD}_{\text {best }}\right)$ from 29 neurons with peak- or trough-shaped tuning measured with static-ITD stimuli plotted against ITD $_{\text {best }}$ measured with dynamic-ITD stimuli. A similar comparison is made for the half-width of ITD tuning in Figure $1 E$ for the same neurons as in Figure $1 D$. Half-width is the range of ITDs between the points where the rate response is $50 \%$ of maximum. Although the best ITDs do not significantly differ for the two stimuli ( $p=0.19$, paired $t$ test), half-widths are significantly narrower with dynamic than with static stimuli $(p<0.005$, paired $t$ test; mean half-width ratio, $0.75 \pm 0.33$ ). Differences between ITD curves measured with these two stimuli may arise from differences in duration of the stimuli and/or the dynamic nature of the ITD. Some neurons showed pronounced adaptation during stimulation with static stimuli, and thus the continuous dynamic stimulus is expected to elicit a lower spike rate. Differences in response to static and dynamic ITD stimuli have also been observed with acoustic stimulation in the IC of normal-hearing animals (Spitzer and Semple, 1991, 1998), where they seem to be consistent with a simple adaptation mechanism dependent on past response history (McAlpine et al., 2000; Borisyuk et al., 2002).

Overall, the differences in ITD tuning for static and dynamic 
A
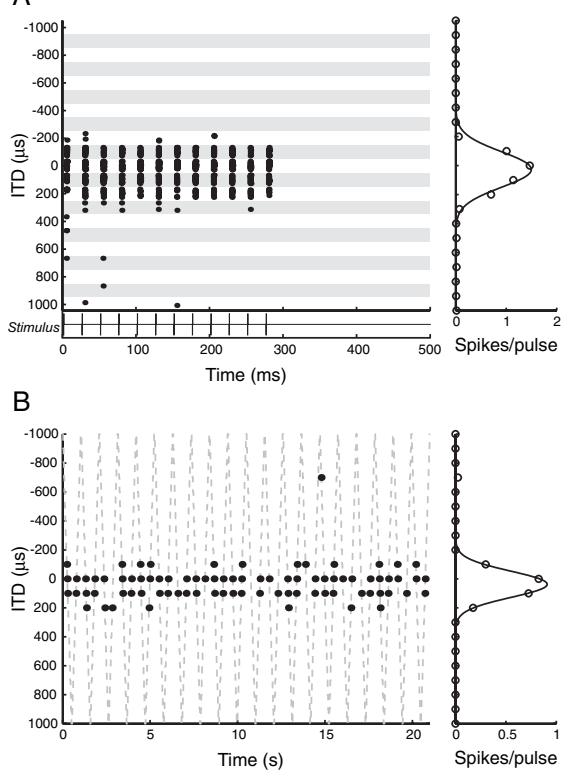

C
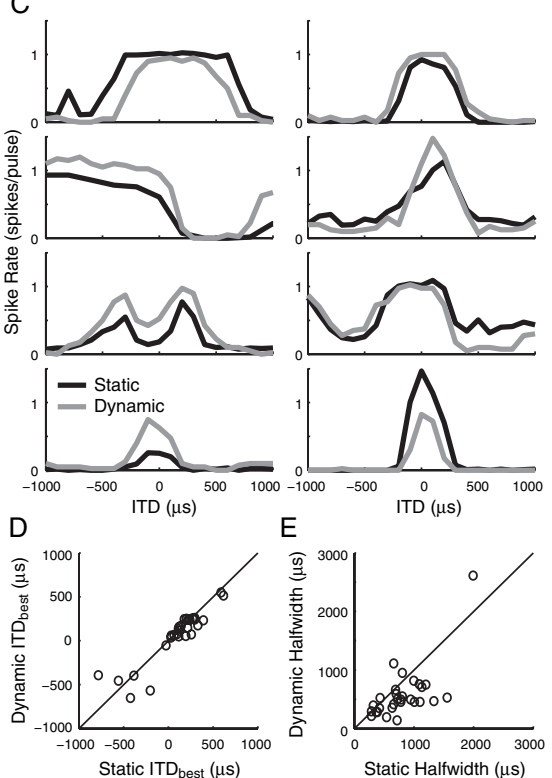

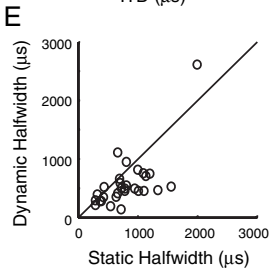

Figure 1. Responses to static- and dynamic-ITD stimuli in the same neurons show similar ITD tuning. $\boldsymbol{A}, \boldsymbol{B}$, Left, Spike raster displays for responses to static and dynamic ITD stimuli in the one neuron. Right, Mean spike rates as a function of ITD. $A$, Response of a neuron to the static-ITD stimulus (stimulus shown under spike raster). Each row in the raster shows the spike time in response to one presentation of a 40 pps pulse train. ITD is varied in blocks of 10 trials indicated by shading. $\boldsymbol{B}$, Response of neuron in $\boldsymbol{A}$ to the dynamic-ITD stimulus. The dashed line shows instantaneous values of ITD as it sweeps periodically between -1000 and $+1000 \mu$ s. The abscissa of each dot indicates the spike time re. stimulus onset, whereas the ordinate indicates the ITD of the immediately preceding stimulus pulse. C, Rate-ITD tuning for static-ITD (black lines) and dynamic-ITD (gray lines) stimuli measured in eight different single neurons. D, Scatter plot of ITD $_{\text {best }}$ in neurons with peak- or trough-shaped ITD tuning for static- and dynamic-ITD stimuli ( $n=29$ neurons). $\boldsymbol{E}$, Scatter plot of half-widths for same neurons as in $\boldsymbol{D}$.

stimuli are relatively minor. Because they provide greater efficiency in data collection, the dynamic ITD stimuli were used both to characterize the shape of ITD tuning and to study how tuning depends on overall current level and ILDs.

\section{Types of ITD tuning}

The rate-ITD curves measured with bilateral electric stimulation with 40 pps pulse trains with dynamic ITDs exhibited a variety of shapes, including peak, trough, monotonic (sigmoid), biphasic, and multipeaked. To quantitatively categorize these shapes, we fit three different mathematical functions to each ITD curve by the least-squares method, and the best-fitting function was used to define the type of ITD tuning (see Materials and Methods). Figure $2 A-D$ shows the mathematical functions characterizing each of the four response types (see Materials and Methods for equations). A fifth group, called "multipeaked," was made up of responses that were not well fit by any of the standard shapes and typically had multiple peaks. Because ITD tuning often changed markedly with intensity, these characterizations were made at a standard intensity, the lowest intensity that elicited a spike rate $>0.5$ spikes/pulse for at least one ITD. This typ- ically occurred 1-2 $\mathrm{dB}$ above threshold. Figure $2 E-H$ shows the rate-ITD curves from all the neurons for each response type (excluding multipeaked). These curves are scaled and shifted so that they are centered at 0 ITD with a width and a height of 1 . The fitted functions clearly capture the shapes of the measured rateITD curves. At the standard intensity, the vast majority of neurons ( 80 of 121) had peak ITD tuning. The ITD tuning of the remaining neurons were roughly evenly split between trough (12 of 121), monotonic (10 of 121), biphasic (9 of 121), and multipeaked (10 of 121) ITD tuning.

\section{Quantitative characterization of ITD tuning}

The fitted curves were used to derive quantitative characterizations of ITD tuning for each neuron in our population using electric stimulation with 40 pps pulse trains at the standard intensity. First, ITD best $_{\text {and }}$ half-width were calculated from Gaussian fits to rate-ITD curves for all peak and trough neurons $(n=92)$. The distribution of $\mathrm{ITD}_{\text {best }}$ is shown as gray bars in Figure $3 A$. The majority ( 68 of 92 ) of ITD $_{\text {best }}$ falls within the natural range of ITD for cat (approximately $\pm 350 \mu \mathrm{s}$ ), and there is a clear contralateral bias to the distribution
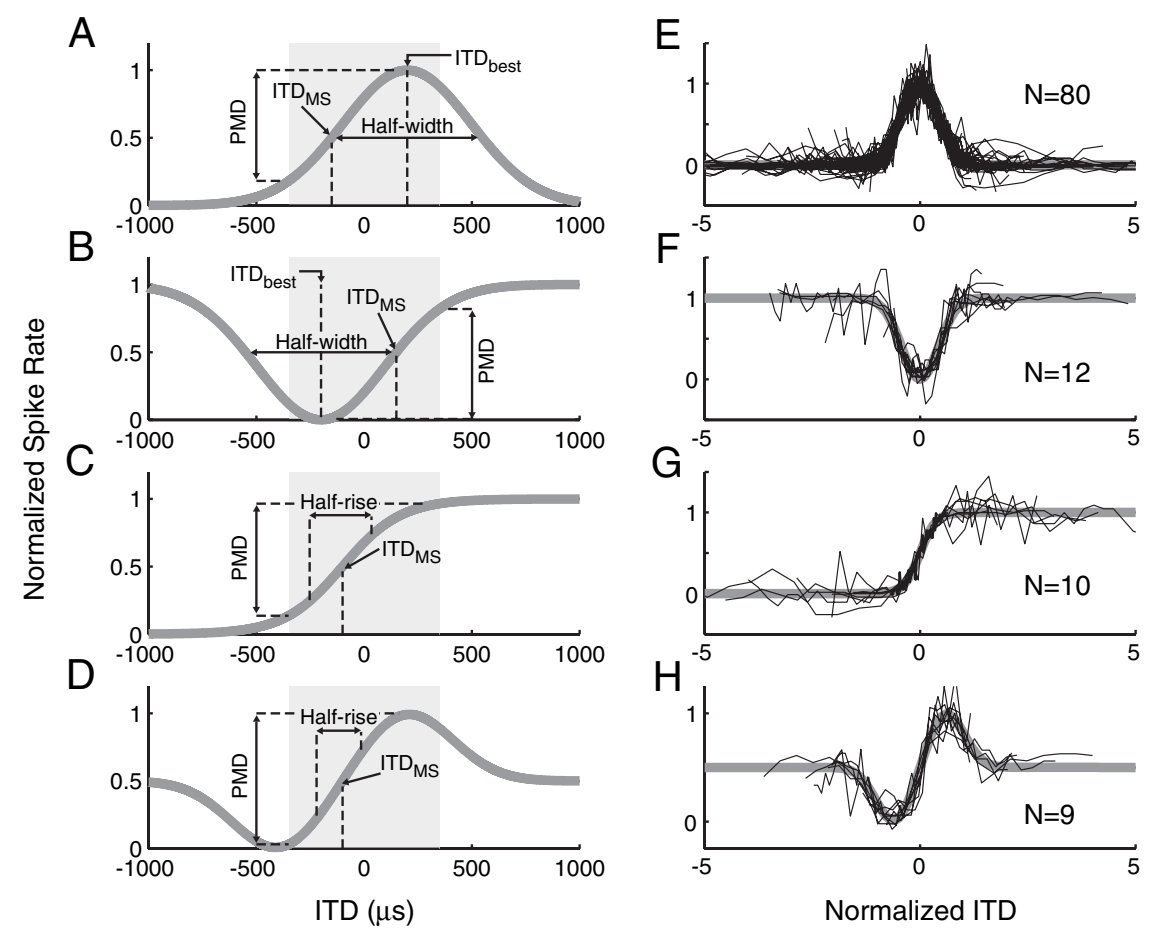

Figure 2. Standard shapes of rate-ITD functions and fits to the data. $\boldsymbol{A}-\boldsymbol{D}$, Idealized response shapes are shown with metrics used to characterize ITD tuning. Shading indicates the range of ITD naturally occurring for a cat. A, A positive Gaussian is used to represent peak-shaped responses. $\boldsymbol{B}$, A negative Gaussian is used to characterize trough-shaped responses. $\boldsymbol{C}$, A sigmoid function is used to characterize monotonic ITD tuning. D, A DOG is used to describe biphasic ITD tuning (see Materials and Methods for equations). $\boldsymbol{E}-\boldsymbol{H}$, Normalized rate-ITD curves of all classified neurons (one trace per neuron) for stimulation with 40 pps pulse trains. Responses are scaled and shifted along both axes to show the goodness of fit of the functions to the data. 
A

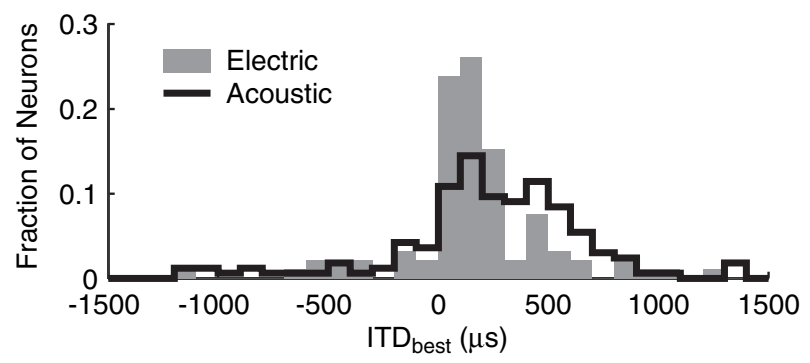

B

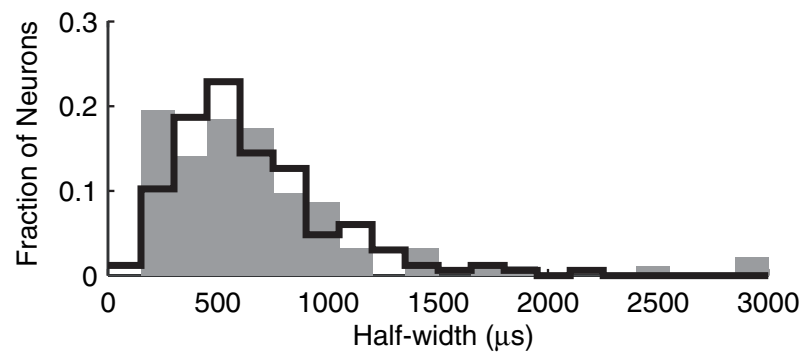

C

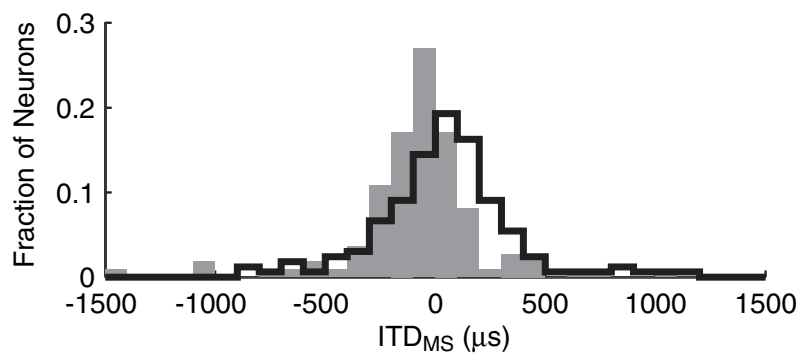

D

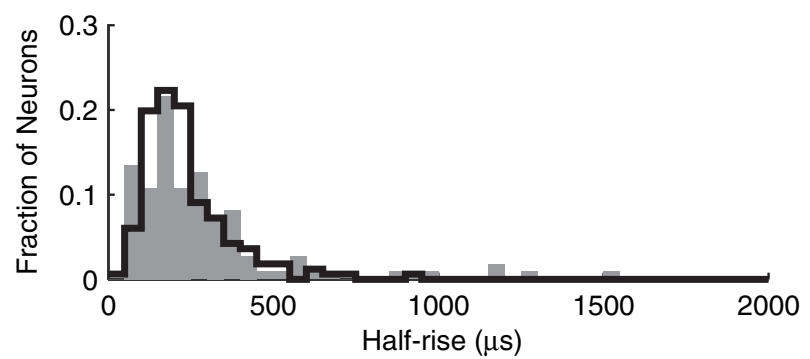

E

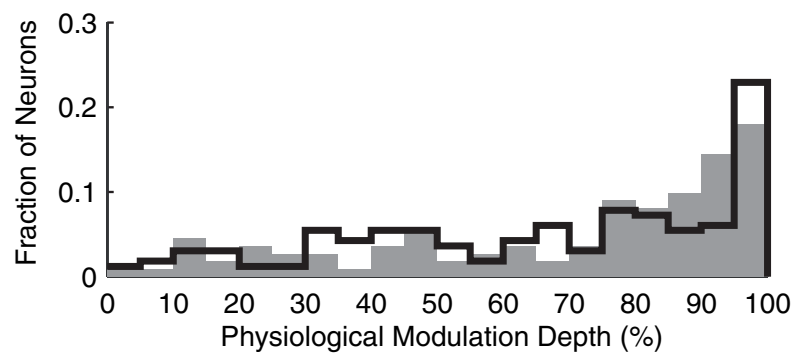

Figure 3. Distributions of $I T D_{\text {best }}$, half-width, $I T D_{M S}$, half-rise, and PMD for both 40 pps electric pulses ( $n=92$ for ITD ${ }_{\text {best }}$ and half-width; $n=111$ for ITD ${ }_{M S}$, half-rise, and PMD) and acoustic broadband noise ( $n=166$ ) (Hancock and Delgutte, 2004).

(mean, $161 \mu \mathrm{s} ; \sigma=335 \mu \mathrm{s} ; 86 \%$ of $\mathrm{ITD}_{\text {best }}>0$ ). For comparison, Figure $3 A$ (black line) also shows ITD $_{\text {best }}$ measured from IC neurons in normal-hearing cats in the same laboratory using stimulation with broadband noise (Hancock and Delgutte, 2004). Although acoustic clicks are more similar to electric pulses than noise, the use of broadband noise for comparison is justified because ITD tuning characteristics are known to be similar for broadband noise and clicks (Carney and Yin, 1989). Both ITD best distributions in Figure $3 A$ have a clear contralateral bias, but the mean $\mathrm{ITD}_{\text {best }}$ for the acoustic data are further from 0 (mean, 252 $\mu \mathrm{s})$, and the spread in the distribution is wider $(\sigma=607 \mu \mathrm{s})$ than for the electric data. A two-sample Kolmogorov-Smirnov (K-S) test shows that $\mathrm{ITD}_{\text {best }}$ distributions for electric and acoustic stimulation are significantly different $(p<0.01)$. This difference may arise in part because the electric population consists of neurons recorded over a wide range of electrode depths, and thus likely to have a wide range of CFs, whereas the acoustic population only contains low-CF neurons $(<3 \mathrm{kHz})$.

The distribution of half-widths for electric stimulation (Fig. $3 B$ ) had a mean of $691 \mu$ s and a pronounced skew toward larger values. Distributions of half-width for electric and acoustic stimulation were not significantly different $(p=0.68$, two-sample $\mathrm{K}-\mathrm{S}$ test). Thus, ITD sensitivity appears to be as sharp with electric pulses as with acoustic broadband noise despite differences in mode of stimulation and neuronal sampling.

Because ITD $_{\text {best }}$ and half-width are only mathematically defined for peak and trough neurons, we also looked at ITD $_{\mathrm{MS}}$, half-rise, and physiological modulation depth (PMD) so as to characterize monotonic and biphasic responses together with the peak and trough. ITD $\mathrm{MS}_{\mathrm{S}}$ is the point at which the greatest change in firing rate occurs for a given change in ITD, and therefore where sensitivity is the greatest. Figure $3 C$ (gray bars) shows the distribution of $\mathrm{ITD}_{\mathrm{MS}}$ for electric stimulation. The $\mathrm{ITD}_{\mathrm{MS}}$ of the majority of neurons (93 of 111) was within the natural range of ITD, with a mean of $-70 \mu$ s and a SD of $311 \mu \mathrm{s}$. The distribution of $\mathrm{ITD}_{\mathrm{MS}}$ for acoustic stimulation (black lines) significantly differs from the electric distribution $(p<0.01$, two-sample K-S test). Indeed, the electric distribution has a slight ipsilateral bias, whereas the acoustic distribution is more closely centered on zero. Also, the electric distribution is narrower than the acoustic distribution, as was the case for $\mathrm{ITD}_{\text {best }}$.

Half-rise measures the range of ITD over which the firing rate rises from 25 to $75 \%$ of its maximum. For peak and trough neurons, half-rise is $\sim 0.4$ times the half-width, on average. The distribution of half-rise for the population of characterized neurons (Fig. 3D) has a mean of $293 \mu$ s and a skew toward higher values. Distributions of electric and acoustic half-rises (gray bars and black lines respectively) were not significantly different ( $p=$ 0.072 , two-sample K-S test), as was the case for half-width. Thus, both metrics suggest that ITD tuning is equally sharp for the two modes of stimulation.

To assess the degree to which neural ITD tuning is significant for naturally occurring stimuli, PMD was calculated from the fits to the rate-ITD curve of each neuron at the standard intensity. PMD is defined as the range of discharge rates within the physiological range of ITD $( \pm 350 \mu \mathrm{s})$ divided by the maximum rate over the entire range of ITD. Figure $3 E$ (gray bars) shows the distribution of PMD for electric stimulation. The mean PMD was 0.70 , and more than half of the neurons had at least an $80 \%$ change in discharge rate within the physiological range of ITD. When compared with acoustic stimulation (black lines), the two distributions are very similar. However, because intensity can have a large effect on PMD with electric stimulation (see below), the similarity between electric and acoustic PMD distributions is likely to depend on the choice of intensity.

Overall, it is remarkable how similar ITD tuning is in IC neurons when stimulated with electric current in the cochlea or with sound pressure at the eardrum. The only significant differences are in the distributions of $\mathrm{ITD}_{\text {best }}$ and $\mathrm{ITD}_{\mathrm{MS}}$, although these differences are small compared with the widths of the distributions. With electric stimulation, neurons tend to have a tighter ITD $_{\text {best }}$ distribution closer to $0 \mu$ s ITD. Because acoustically $\mathrm{ITD}_{\text {best }}$ inversely correlates with CF, differences between acoustic 

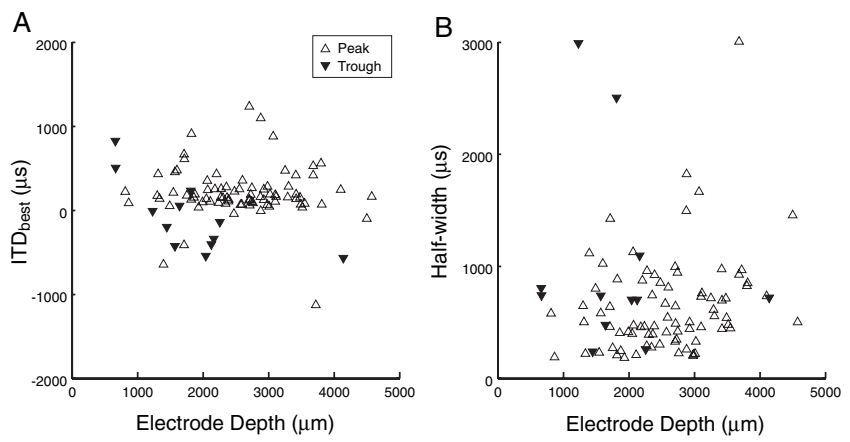

Figure 4. ITD $\mathrm{IDest}_{(\boldsymbol{A})}$ and ITD tuning half-width $(\boldsymbol{B})$ as a function of electrode depth along the tonotopic axis of IC.

and electric ITD tuning may be a consequence of recording from a wider range of CFs in the present study than in the acoustic study from the same laboratory (Hancock and Delgutte, 2004). We test this idea next.

\section{Dependence on electrode depth along tonotopic axis}

Since CF cannot be directly measured in neurons from deafened animals, ITD tuning characteristics were analyzed as a function of electrode depth, which closely correlates with CF in our dorsoventral electrode penetrations (Snyder et al., 1990). The electrode penetrations were either tilted $45^{\circ}$ off the sagittal plane (standard trajectory) or were straight vertical (vertical trajectory). The standard angle was chosen so that the electrode would travel parallel to the tonotopic axis of the ICC. The CF of the neurons increases with increasing electrode depth (Merzenich and Reid, 1974; Snyder et al., 1990), with low-CF neurons at the dorsolateral edge of the ICC and high-CF neurons at deeper depths (toward ventromedial). Although CF is expected to increase with distance at a slower rate for the vertical trajectory than for the standard trajectory $\left(45^{\circ}\right)$, separate analyses did not reveal any obvious difference between the two trajectories, so the two sets of data were combined.

Figure 4 shows ITD $_{\text {best }}$ and half-width as a function of electrode depth for peak and trough responses. With acoustic stimulation, both $\mathrm{ITD}_{\text {best }}$ and half-width are inversely correlated with CF in low-frequency ITD-sensitive IC neurons (McAlpine et al., 2001). In contrast, there is no obvious trend for $\mathrm{ITD}_{\text {best }}$ and halfwidth with IC depth for electric stimulation, although the ITD $_{M S}$ distribution is centered near 0 for both modes of stimulation (Fig. $3 C)$. Statistical regression analyses revealed no significant correlation of either $\mathrm{ITD}_{\text {best }}$, half-width, $\mathrm{ITD}_{\mathrm{MS}}$, half-rise, or PMD with electrode depth $\left(r^{2}<0.01\right.$ and $p>0.35$ for all metrics $)$.

\section{Effect of overall intensity}

Thus far, all rate-ITD responses were measured at the standard intensity, which was usually $1-2 \mathrm{~dB}$ above threshold. Many neurons were studied over a range of intensities using dynamic ITD stimuli, and we found that stimulus intensity could have a large effect on rate-ITD tuning. Figure 5 shows rate-ITD curves for nine neurons over a range of intensities. The three neurons shown in the top row had peak-shaped ITD tuning at levels just above threshold, but, as intensity increased, their ITD tuning broadened and eventually saturated at $7,1.5$, and $2.6 \mathrm{~dB}$ above threshold, respectively. The middle row shows rate-ITD curves for three more neurons that had peak-shaped tuning at low intensities that broadened with increasing intensity. However, unlike the neurons in the top row, these neurons did not fully satu- rate at any of the intensities tested. The bottom row shows three neurons that showed little or no degradation in ITD tuning with increasing intensity over the range tested. In fact, tuning for the trough response in the bottom middle panel improved with increasing intensity.

For many neurons, changes in stimulus intensity altered the shape of ITD tuning. For example, the ITD tuning of the neuron shown in the top left plot of Figure 5 was peak shaped at $2 \mathrm{~dB}$ above threshold but changed into trough shaped at $6 \mathrm{~dB}$ above threshold. At an even higher intensity $(7 \mathrm{~dB})$, the ITD tuning of this neuron saturated. Table 1 lists the distributions of ITD tuning shapes for 99 neurons that were studied at a minimum of three intensities. At the lowest intensities (mean level, $1.3 \pm 1.1$ $\mathrm{dB}$ re. threshold), most neurons (75 of 99) had peak responses. At intermediate levels $(2.5 \pm 1.1 \mathrm{~dB}$ re. threshold $)$, the number of peak responses decreased to 42 neurons, whereas the number of biphasic responses increased from 8 to 29 of 99 neurons, and the proportions of trough- and monotonic-shaped neurons also increased somewhat. At the highest levels $(3.6 \pm 1.7 \mathrm{~dB}$ re. threshold), there was an additional decrease in the number of peak responses and a major increase in saturated responses (from 5 to 17 of 99 neurons). These changes may reflect the increasing role of saturation in determining ITD tuning shapes at higher levels.

A direct analogy with results from normal-hearing animals would suggest that peak-type responses may reflect predominant input from MSO neurons showing excitatory/excitatory binaural interactions (Goldberg and Brown, 1969; Yin and Chan, 1990), whereas trough responses would reflect inputs from excitatory/ inhibitory neurons in the lateral superior olive (Finlayson and Caspary, 1991; Tollin and Yin, 2005). The finding that the type of ITD curve often changes with level, the lack of a systematic gradient in response types along the tonotopic axis of the IC (Fig. 4), and model simulations showing that the response of a simple coincidence detector can change from peak type to trough type as the instantaneous firing rate of the inputs increases (Brughera et al., 1996) suggest that this interpretation is oversimplified. It is likely that factors specific to electric stimulation, such as the high rates of discharge and increased synchrony at the level of the auditory nerve (Kiang and Moxon, 1972; Hartmann et al., 1984), and the downregulation of inhibition resulting from deafening (Kitzes and Semple, 1985; Vale and Sanes, 2002) play an important role in shaping the types of ITD tuning observed.

\section{Effect of ILDs}

Because naturally occurring sound sources give rise to interaural differences in intensity as well as in time, the effect of ILD on ITD tuning was studied for some cells. ILD was varied by changing the current level in both ears by equal steps in opposite directions, thereby maintaining a fixed MBL. Typically, within a small range of ILD, ITD tuning remained well defined, but there were systematic shifts in $\mathrm{ITD}_{\text {best }}$. Figure $6 \mathrm{~A}$ shows normalized rate-ITD curves of a unit for ILDs ranging from -3 to $+3 \mathrm{~dB}$. Although tuning width remains nearly constant, $\mathrm{ITD}_{\text {best }}$ shifts toward more contralateral leading values (positive ITD) with increasing intensity in the contralateral ear (Fig. $6 \mathrm{~B}$ ). A straight line fit to the data has a slope of $\sim 60 \mu \mathrm{s} / \mathrm{dB}$ and is used to express changes in $\mathrm{ITD}_{\text {best }}$ as a time-intensity trading ratio $\left(\Delta \mathrm{ITD}_{\text {best }} / \Delta \mathrm{ILD}\right)$. Figure $6 C$ shows ITD $_{\text {best }}$ versus ILD for 16 units that had peak-shaped ITD tuning and were tested at multiple ILDs. Eleven units had negative time-intensity trading ratios, which are consistent with psychophysical trading ratios (David et al., 1959), whereas 5 of 16 units had positive ratios. The average magnitude of the trading ratios was $42 \mu \mathrm{s} / \mathrm{dB}$. 
Time-intensity trading ratios have been measured in the IC with acoustic stimulation in normal-hearing animals. Yin and Kuwada (1983), using pure tone stimuli in low-CF neurons, found mean trading ratios magnitudes of $5.8 \mu \mathrm{s} / \mathrm{dB}$, with over two-thirds of the ratios being positive. A study of high-CF neurons (Caird and Klinke, 1987) reported a mean magnitude of time-intensity trading ratios of 91 $\mu \mathrm{s} / \mathrm{dB}$ with $\mathrm{CF}$-tone stimulation and 40 $\mu \mathrm{s} / \mathrm{dB}$ with click stimuli, although the signs of the trading ratios were not reported. Our results for electric stimulation with pulse trains are most consistent with acoustic stimulation of high-CF neurons with clicks. However, it is difficult to directly compare trading ratios for acoustic and electric stimulation because the electric dynamic range is much smaller than the acoustic dynamic range (both neurally and behaviorally) and because cochlear implant processors introduce severe compression to compensate for the reduced electric dynamic range.

\section{Neural discrimination thresholds}

So far, we have reported responses to 40 pps pulse trains with either static or dynamic ITD. In a few neurons, we also examined ITD tuning for a range of pulse rates (40-320 pps) to compare the dependence of neural responses on pulse rate with that of ITD discrimination performance in bilaterally implanted human subjects. We used static ITD stimuli for this purpose because the short interpulse intervals at higher pulse rates make an unambiguous assignment of each spike to a given ITD problematic for dynamic stimuli. Temporal discharge patterns from an example neuron are shown as a function of ITD for four pulse rates in Figure 7A. Although spikes have an almost one-to-one relationship to each stimulus pulse for low pulse rates at favorable ITDs, responses become increasingly limited to the stimulus onset at higher pulse rates, resulting in a marked decrease in overall firing rate. The shape of ITD tuning curves is maintained at higher stimulation rates despite this decrease in firing rate (Fig. $7 B)$.

In an effort to compare ITD sensitivity with electric cochlear stimulation between single neurons in our cat preparation and human behavior, neural detection thresholds were estimated using a metric from signal detection theory. We computed the standard separation (Sakitt, 1973) between each ITD step and a reference ITD of $0 \mu \mathrm{s}$. This analysis was used to estimate the detection threshold in single units based on the spike-count statistics of individual trials. Standard separation is plotted as a function of ITD for the example neuron in Figure 7C. The neural JND is the ITD closest to 0 for which the standard separation is 1 (dashed line). For the neuron in Figure 7C, the ITD JNDs at 40, of at least three intensities.
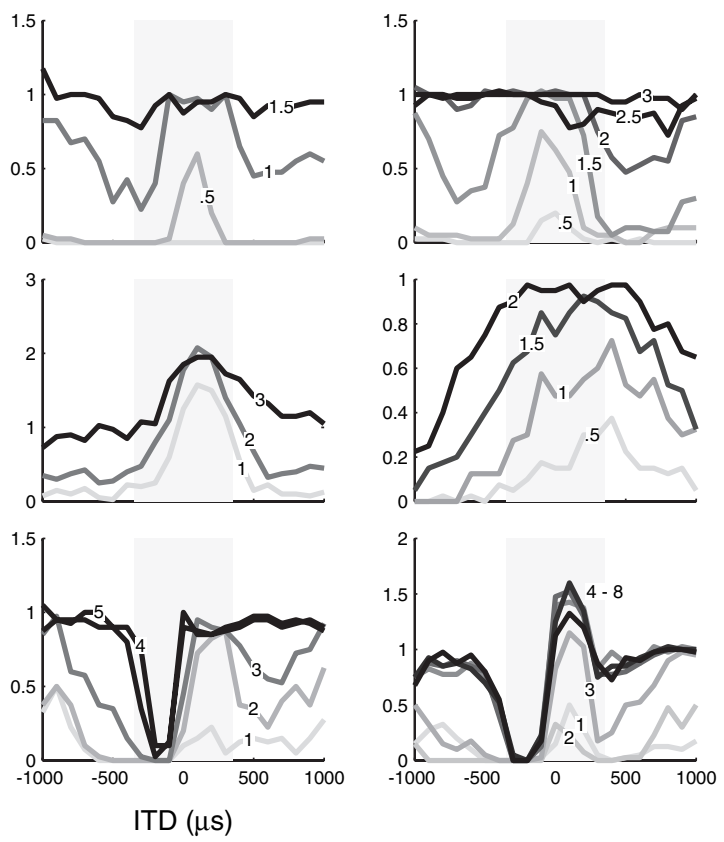

Figure 5. Effect of stimulus intensity on ITD tuning. ITD tuning for nine neurons at a range of stimulus intensities. Numbers on curves indicate intensity in decibels re. threshold.

Table 1. Distribution of ITD tuning shapes at different stimulus levels

\begin{tabular}{lccc}
\hline ITD tuning shape & Low levels $(1.3 \pm 1.1 \mathrm{~dB})$ & Mid levels $(2.5 \pm 1.1 \mathrm{~dB})$ & High levels $(3.6 \pm 1.7 \mathrm{~dB})$ \\
\hline Peak & 75 & 42 & 31 \\
Trough & 12 & 15 & 15 \\
Monophasic & 4 & 8 & 11 \\
Biphasic & 8 & 29 & 25 \\
Saturated & 0 & 5 & 17
\end{tabular}

The mean level \pm 1 SD is given for each level group in units of decibels re. threshold. Data are the numbers of neurons of a total of 99 neurons tested at a range
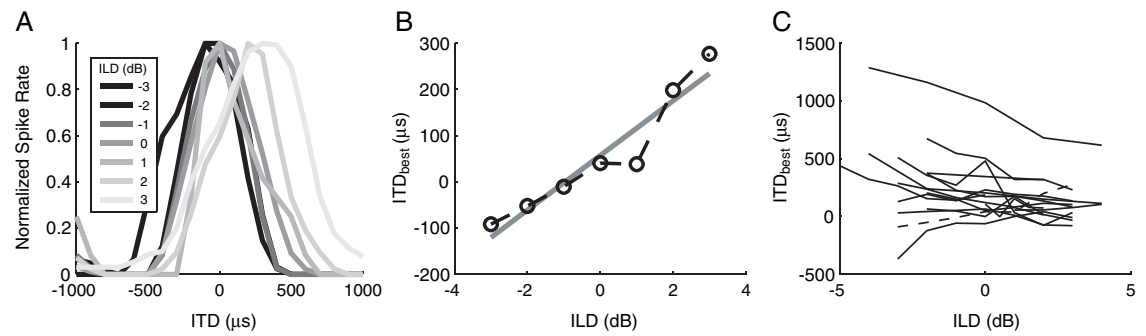

Figure 6. Effect of ILD on ITD tuning. $\boldsymbol{A}$, ITD tuning in a single neuron for seven different ILDs. $\boldsymbol{B}$, ITD $_{\text {best }}$ as a function of ILD for the neuron in $\boldsymbol{A}$. $\boldsymbol{C}, \mathrm{ITD}_{\text {best }}$ as a function of ILD for 16 neurons. The neuron from $\boldsymbol{A}$ is indicated with a dashed line. Eleven of 16 functions have negative slopes when fit with a straight line.

80,160 , and 320 pps are $118,250,460$, and $620 \mu$ s, respectively (arrows). For all four pulse rates, the lowest JND occurs when the ipsilateral ear leads (negative ITDs), as typically occurs for acoustic stimulation in normal-hearing animals (Shackleton et al., 2003).

Figure $8 \mathrm{~A}$ shows the mean ITD discrimination thresholds as a function of pulse rate for eight neurons. At 40 and 80 pps, mean neural ITD JNDs were $\sim 125 \mu \mathrm{s}$. At higher stimulus rates, the ITD needed to produce a significant change in the firing rate of a neuron increases. The mean ITD JNDs at 160 and 320 pps were 225 and $375 \mu$ s, respectively.

To compare neural ITD JNDs with human behavioral data for pulse trains of different durations, we characterized the relative 
40 pps

80 pps

$160 \mathrm{pps}$

320 pps
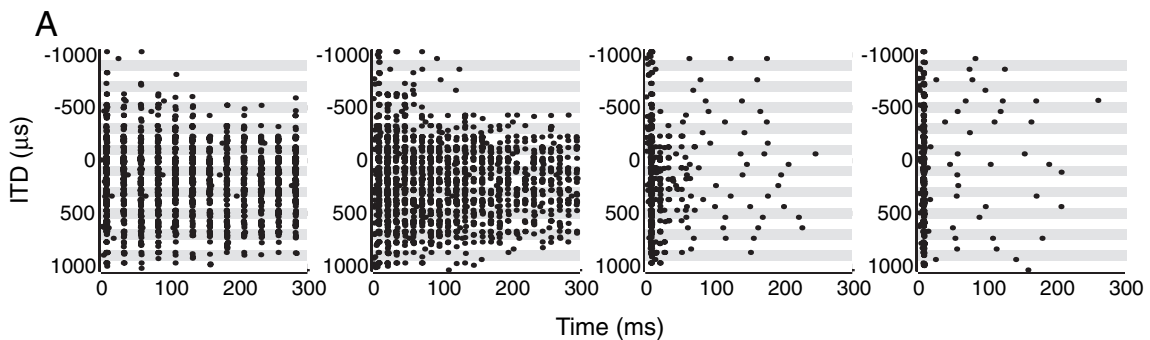

B
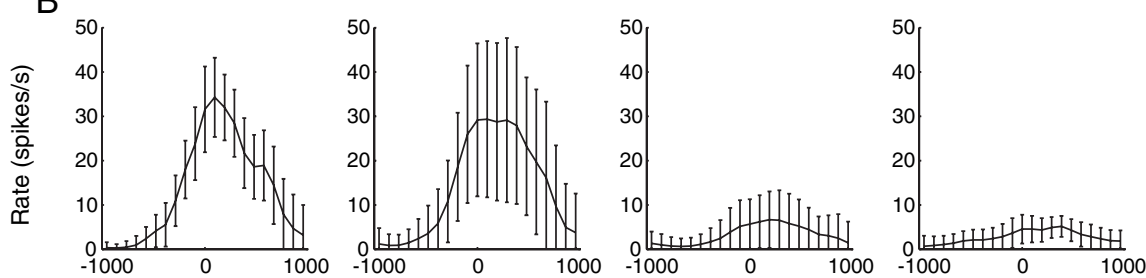

ITD $(\mu \mathrm{s})$
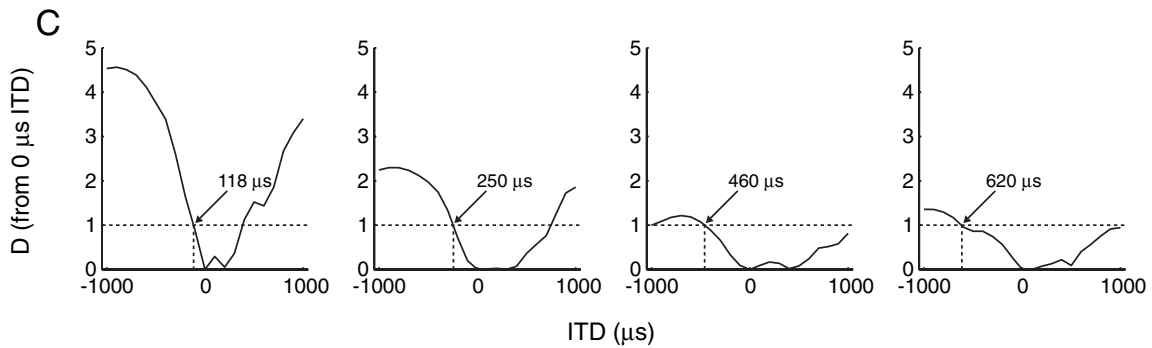

Figure 7. ITD sensitivity of a neuron for four different pulse rates. $A$, Dot-raster display shows temporal discharge patterns to pulse train stimulus as a function of ITD for different rates. At higher rates, the response is mostly limited to the stimulus onset (stimulus duration is $300 \mathrm{~ms}$ ). B, Rate-ITD curves for each stimulus rate. Error bars show \pm 1 SD over stimulus presentations. $\boldsymbol{C}$ Standard separation (D) from a reference of $0 \mu$ S ITD as a function of ITD. ITD JND (arrow) is where D $=1$ (dotted horizontal line). for 12 pulses. The slope of the best-fitting line was approximately -0.5 (inverse square root), which is the theoretical improvement predicted by perfect integration of equal ITD information from each pulse in the train. The dashed line in Figure $8 C$ shows the best neural ITD JND among the 22 neurons tested as a function of the number of pulses. The best neural thresholds were approximately five times smaller (better) than the mean thresholds, decreasing from $\sim 100 \mu$ s for one pulse to $\sim 20 \mu$ s for 12 pulses.

All of the ITD discrimination thresholds up to this point were calculated from a reference ITD of $0 \mu \mathrm{s}$. Figure $8 D$ plots the mean ITD JND at 40 pps as a function of reference ITD ( $n=22$ neurons). The mean neural JNDs are best between reference ITDs of -300 to $+300 \mu$ s and degrade as the reference ITDs increasingly deviates from this range. This result is consistent with the finding presented in Figure $3 C$ that the distribution of ITD $_{\mathrm{MS}}$ is centered near $0 \mu$ s ITD and has a SD of $\sim 300$ $\mu \mathrm{s}$.

\section{Discussion}

This is the first single-unit neurophysiological study of ITD sensitivity with bilateral cochlear implants. Our main finding is that ITD sensitivity of IC neurons with electric stimulation of the cochlea in acutely deafened animals is, in many ways, contribution of onset and sustained responses at different pulse rates by splitting responses into two time segments and calculating the ITD JND for each segment separately. Mean discrimination thresholds based on the first $50 \mathrm{~ms}$ of the response ("onset") and the remaining $250 \mathrm{~ms}$ of the response ("sustained") are plotted together with mean thresholds based on the entire $300-\mathrm{ms}$ response in Figure 8 B. Thresholds based on the onset response do not vary much with pulse rate, whereas thresholds from the sustained response are better than the onset thresholds at 40-80 pps but rapidly get worse and exceed the natural range of ITDs at 160 and 320 pps. Comparison of thresholds based on the onset and sustained responses with those based on the entire response shows that, at $40 \mathrm{pps}$, thresholds are dominated by the sustained response, whereas at 160 and 320 pps, thresholds are dominated by the onset response. This is consistent with results in normalhearing animals that show that increasing the stimulus duration improves neural ITD JNDs in IC neurons with sustained responses but not in neurons with onset or pauser responses (Shackleton et al., 2003).

Because sustained responses are primarily responsible for the good ITD discrimination observed with low-rate pulse trains, we also analyzed the contribution of each pulse in a $40 \mathrm{pps}$ train to the discrimination threshold. Because spikes are tightly locked to each stimulus pulse, this was achieved by separately windowing the response to each pulse. Figure $8 C$ shows ITD JNDs for 22 neurons as a function of the number of pulses in a 40 pps pulse train. Mean ITD discrimination thresholds dropped linearly on a $\log -\log$ scale, decreasing from $\sim 500 \mu$ s for one pulse to $\sim 100 \mu \mathrm{s}$
A

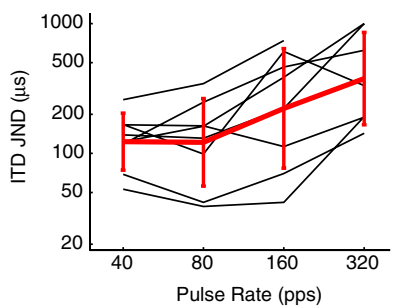

C

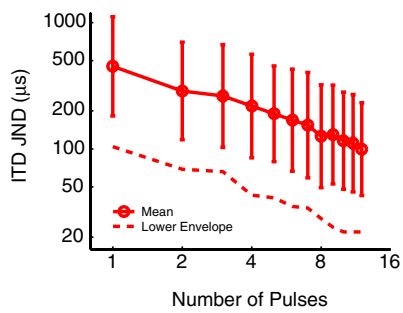

D

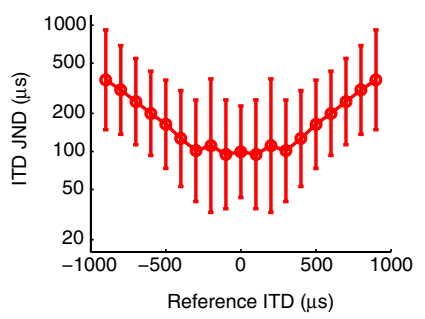

Figure 8. Neural ITD discrimination thresholds. $A$, ITD JND from $0 \mu$ s is as a function of pulse rate for eight single neurons (thin black lines). Mean JND across neurons increases with pulse rate (thick red line). Error bars show $\pm 1 S D$. B, The first $50 \mathrm{~ms}$ of the response (onset response) is analyzed separately from the remaining $250 \mathrm{~ms}$ of the response (sustained response). C, ITD thresholds as a function of the number of stimulus pulses in a 40 pps pulse train. Mean ITD JNDs drop linearly on a $\log -\log$ scale. Error bars show $\pm 1 S D$. The dotted line shows the lower envelope of the ITD thresholds from individual neurons $(n=22)$. $D$, Neural ITD JNDs as a function of reference ITD. Mean thresholds are best within the physiological range of ITD ( \pm 300 $\mu \mathrm{s}$ ) and get worse at increasing ITD offsets. 
similar to that with acoustic stimulation in normal-hearing animals despite the lack of cochlear processing with electric stimulation. This result is encouraging for the prospect of restoring the benefits of binaural processing based on ITD to bilaterally implanted individuals.

\section{Comparison of ITD tuning for electric and acoustic stimulation}

We compared ITD tuning for electric stimulation, recorded from neurons likely to cover a wide range of CFs, with acoustic data from low-CF neurons in normal-hearing animals. This sampling difference in CF may partly explain the observed differences in the distributions of ITD $_{\text {best }}$ and ITD $_{\mathrm{MS}}$ with acoustic and electric stimulation (Fig. $3 A, C$ ), although there was no evidence for a dependence of these parameters on electrode depth in our data (Fig. 4). Despite the different sampling of neurons, the distributions of half-width, half-rise, and PMD found with electric stimulation are remarkably similar to those found in low-CF neurons using acoustic stimulation with broadband noise (Fig. $3 B, D, E$ ). For noise stimuli, both $\mathrm{ITD}_{\text {best }}$ and half-width are inversely correlated with CF in the IC of normal-hearing animals (McAlpine et al., 2001; Hancock and Delgutte, 2004; Joris et al., 2006). These trends are not observed with pulsatile electric stimulation (Fig. 4 ), possibly as a result of bypassing cochlear filtering. It has been suggested that the internal delays underlying neural best ITD may arise from interaural disparities in the CFs of the inputs to the coincidence detector neurons in the MSO and therefore differences in delays of the cochlear traveling waves (Schroeder, 1977; Shamma et al., 1989; Joris et al., 2006). The different relationships between $\mathrm{ITD}_{\text {best }}$ and $\mathrm{CF}$ observed for acoustic versus electric stimulation lend some support for these views. However, the observation of a contralateral bias in the distributions of ITD $_{\text {best }}$ for both modes of stimulation suggests that the delays cannot be entirely accounted for by the cochlear-traveling waves and may result from other mechanisms such as axonal conduction delay (Jeffress, 1948; Smith et al., 1991) and/or inhibition (Brand et al., 2002).

ITD tuning characteristics were remarkably homogenous with electric stimulation, regardless of the electrode depth along the tonotopic axis of the IC. This suggests that frequencydependent differences in binaural processing seen with acoustic hearing, such as broader ITD tuning and loss of sensitivity to fine-structure ITD in high-CF neurons, may be primarily influenced by differences in cochlear processing and not by differences in brainstem neural mechanisms (Colburn and Esquissaud, 1976). This view is also supported by psychophysical and physiological studies using "transposed stimuli" (Bernstein and Trahiotis, 2002; Griffin et al., 2005). Whereas the sharpness of neural ITD tuning is limited by cochlear frequency tuning with broadband acoustic stimulation (Yin et al., 1986), this is not the case for electric stimulation, in which cochlear filtering is bypassed and the sharpness of ITD tuning may reflect the combined effects of the width of the coincidence window in binaural neurons and temporal jitter in the inputs to these neurons. Thus, the finding that ITD tuning is no better with electric stimulation than with acoustic stimulation, despite eliminating the additional limitation imposed by cochlear filtering, suggests that either we may be sampling in part from a broader population of neurons with electric stimulation, or the ITD processing mechanisms may not operate as efficiently with electric stimulation as in normal hearing. Possible reasons for such inefficiency would be the abnormally high synchronous firing of the inputs to the binaural neu- rons and distortions in the normal spatiotemporal patterns of these inputs.

ITD tuning was found to be highly sensitive to overall stimulus intensity with electric stimulation. Intensity steps as small as 1 $\mathrm{dB}$ were sometimes sufficient to dramatically change the shape and reduce the tuning of rate-ITD curves in individual neurons (Fig. 5). In contrast, stimulation with low-frequency acoustic tones shows relatively robust ITD tuning over $40 \mathrm{~dB}$, with only slight shifts in ITD $_{\text {best }}$ (Kuwada and Yin, 1983; Fitzpatrick et al., 2005). This difference between acoustic and electric stimulation may arise as early as the auditory nerve, where the dynamic range of single-fiber rate responses is $\sim 15-40 \mathrm{~dB}$ for acoustic pure tones and noise (Sachs and Abbas, 1974), whereas it does not exceed a few decibels for low-rate electric pulse trains and sinusoids (Moxon, 1967; van den Honert and Stypulkowski, 1987; Javel and Shepherd, 2000). It is difficult to assess the impact of this small neural dynamic range on behavioral ITD sensitivity. A restricted dynamic range $(10-20 \mathrm{~dB})$ of ITD tuning has been reported in IC neurons with clicks, an acoustic stimulus that resembles our current pulses (Carney and Yin, 1989). However, clicks are among the stimuli that give the best behavioral ITD discrimination in normal-hearing humans (Klumpp and Eady, 1956; Yost et al., 1971), suggesting that a large dynamic range in single neurons may not be behaviorally essential. Behavioral performance may be based either on a subpopulation of IC neurons with relatively wide dynamic ranges (Fig. 5, bottom row) or on a level-dependent subpopulation of neurons, which individually only have good ITD tuning over a narrow intensity range but together cover a wide dynamic range.

\section{Effect of pulse rate on IC responses}

Neural ITD JNDs generally were best for low pulse rates $(<100$ pps) and degraded rapidly with increasing pulse rate (Figs. 7 , $8 A, B)$ as responses became mostly limited to the onset of the pulse train. A similar inability of IC neurons to give sustained responses at frequencies above $100-300 \mathrm{~Hz}$ has been reported with monolateral electric stimulation with pulse trains and sinusoids in anesthetized animals (Snyder et al., 1991, 1995) but is not consistently observed for stimulation with pure tones in nomalhearing animals, in which about half of high-CF neurons show sustained responses even at tens of kilohertz (Le Beau et al., 1996; Nuding et al., 1999). Sustained responses are also observed in the auditory nerve for high-frequency electric stimulation, although some fibers show pronounced adaptation (Moxon, 1967; van den Honert and Stypulkowski, 1987; Parkins, 1989; Litvak et al., 2003). Suprathreshold electric stimulation at rates below 1000 pps often elicits entrained spikes in auditory nerve fibers (one spike for each current pulse), which does not occur for acoustic stimulation. If IPSPs are relatively slow, then such entrainment may result in inhibition gradually building up over time. The lack of sustained responses in the IC at high rates with electric stimulation may result from such a buildup of inhibition.

\section{Comparison of neural ITD discrimination with human psychophysics}

Substantial intersubject variability in human psychophysical data with bilateral cochlear implants makes it difficult to directly compare these data with ITD JNDs for single IC neurons. Nevertheless, there are clear parallels in the dependence of neural and behavioral JNDs on several stimulus parameters. Neural ITD JNDs generally degraded with increasing pulse rates above 100 pps (up to 320 pps tested) (Fig. 8A). This trend has also been observed psychophysically in human subjects, although the 
sharpest increases in JNDs seem to occur at higher pulse rates (van Hoesel and Tyler, 2003; Poon, 2006; van Hoesel, 2007). By separately analyzing different time segments of the neural response, we showed that, at lower pulse rates ( $<100 \mathrm{pps}$ ), the ITD JNDs are dominated by information provided by the sustained portion of the response (Fig. $8 \mathrm{~B}$ ), whereas at the highest rate tested ( $320 \mathrm{pps}$ ), only the onset response contributed to the ITD JND. Similarly, increasing the stimulus duration improves behavioral ITD discrimination thresholds for lower-rate pulse trains but not higher-rate pulse trains (Poon, 2006; van Hoesel, 2007). Finally, neural ITD JNDs for 40 pps pulse trains dropped (improved) in inverse proportion to the square root of the number of pulses in the train (Fig. $8 C$ ). The same trend, which indicates optimal integration of equal ITD information from each pulse in the train, has also been observed with 50-100 pps pulse trains in the human behavioral data (Poon, 2006; van Hoesel, 2007).

It is remarkable that these trends in ITD discrimination are so similar between the physiology and psychophysics despite many differences between our animal model and human subjects. There are also many similarities in the ITD tuning seen in the IC with electric and acoustic stimulation. Despite these similarities, there is still a large gap in ITD discrimination performance of normal-hearing and bilaterally implanted human subjects. The best neural ITD JNDs were on the order of $20 \mu \mathrm{s}$, whereas ITD JNDs in the best human subjects are rarely below $100 \mu$ s and are much larger in most subjects. This performance gap is observed even when the clinical sound processors used on a daily basis by cochlear implant wearers is bypassed in psychophysical experiments. Most processors do not preserve the fine time structure of the acoustic signal normally important for precise ITD discrimination (Wightman and Kistler, 1992; Macpherson and Middlebrooks, 2002). The acutely deafened animals used in this study represent the best-case scenario in terms of neural survival and normal binaural experience. On the contrary, the human subjects tested in the behavioral studies have varying amounts of binaural experience both before the onset of deafness and with bilateral implants and also differ in duration of deafness and neural survival. These differences in experience may underlie the disparity in absolute ITD discrimination thresholds between acoustic and electric hearing. Future neurophysiological studies need to address the issues of experience and neural plasticity on binaural processing with cochlear implants.

\section{References}

Aitkin L, Schuck D (1985) Low frequency neurons in the lateral central nucleus of the cat inferior colliculus receive their input predominantly from the medial superior olive. Hear Res 17:87-93.

Bernstein LR, Trahiotis C (2002) Enhancing sensitivity to interaural delays at high frequencies by using "transposed stimuli." J Acoust Soc Am 112:1026-1036.

Blauert J (1997) Spatial hearing: the psychophysics of human sound localization, revised edition. Cambridge, MA: MIT.

Borisyuk A, Semple MN, Rinzel J (2002) Adaptation and inhibition underlie responses to time-varying interaural phase cues in a model of inferior colliculus neurons. J Neurophysiol 88:2134-2146.

Brand A, Behrend O, Marquardt T, McAlpine D, Grothe B (2002) Precise inhibition is essential for microsecond interaural time difference coding. Nature 417:543-547.

Brughera A, Stutman E, Carney L, Colburn H (1996) A model for excitation and inhibition for cells in the medial superior olive. Audit Neurosci 2:219-233.

Caird D, Klinke R (1987) Processing of interaural time and intensity differences in the cat inferior colliculus. Exp Brain Res 68:379-392.

Carney LH, Yin TC (1989) Responses of low-frequency cells in the inferior colliculus to interaural time differences of clicks: excitatory and inhibitory components. J Neurophysiol 62:144-161.

Colburn HS, Esquissaud P (1976) An auditory-nerve model for interaural time discrimination of high-frequency complex stimuli. J Acoust Soc Am Suppl 59:S23.

David EE, Gurttman N, van Bergeijk WA (1959) Binaural interaction of high-frequency complex stimuli. J Acoust Soc Am 31:139-145.

Delgutte B, Joris PX, Litovsky RY, Yin TC (1999) Receptive fields and binaural interactions for virtual-space stimuli in the cat inferior colliculus. J Neurophysiol 81:2833-2851.

Durlach NI, Colburn HS (1978) Binaural phenomena. In: Handbook of perception (Carterette E, Friedman MP, eds), pp 365-466. New York: Academic.

Finlayson PG, Caspary DM (1991) Low-frequency neurons in the lateral superior olive exhibit phase-sensitive binaural inhibition. J Neurophysiol 65:598-605.

Fitzpatrick DC, Patel MR, Ebert CS, Demke JC (2005) The effect of intensity on ITD tuning to pure tones. Paper presented at Association for Research in Otolaryngology Midwinter Meeting, New Orleans, LA, February.

Fu QJ, Shannon RV, Wang X (1998) Effects of noise and spectral resolution on vowel and consonant recognition: acoustic and electric hearing. J Acoust Soc Am 104:3586-3596.

Goldberg JM, Brown PB (1969) Response of binaural neurons of dog superior olivary complex to dichotic tonal stimuli: some physiological mechanisms of sound localization. J Neurophysiol 32:613-636.

Green DM, Swets JA (1966) Signal detection theory and psychophysics. New York: Krieger.

Griffin SJ, Bernstein LR, Ingham NJ, McAlpine D (2005) Neural sensitivity to interaural envelope delays in the inferior colliculus of the guinea pig. J Neurophysiol 93:3463-3478.

Hancock KE, Delgutte B (2004) A physiologically based model of interaural time difference discrimination. J Neurosci 24:7110-7117.

Hartmann R, Topp G, Klinke R (1984) Discharge patterns of cat primary auditory fibers with electrical stimulation of the cochlea. Hear Res $13: 47-62$.

Hochberg I, Boothroyd A, Weiss M, Hellman S (1992) Effects of noise and noise suppression on speech perception by cochlear implant users. Ear Hear 13:263-271.

Irvine DR, Gago G (1990) Binaural interaction in high-frequency neurons in inferior colliculus of the cat: effects of variations in sound pressure level on sensitivity to interaural intensity differences. J Neurophysiol 63:570-591.

Javel E, Shepherd RK (2000) Electrical stimulation of the auditory nerve. III. Response initiation sites and temporal fine structure. Hear Res 140:45-76.

Jeffress LA (1948) A place theory of sound localization. J Comp Physiol Psychol 41:35-39.

Joris PX, Van de Sande B, Louage DH, van der Heijden M (2006) Binaural and cochlear disparities. Proc Natl Acad Sci USA 103:12917-12922.

Kiang NYS, Moxon EC (1972) Physiological considerations in artificial stimulation of the inner ear. Ann Otol Rhinol Laryngol 81:714.

Kitzes LM, Semple MN (1985) Single-unit responses in the inferior colliculus: effects of neonatal unilateral cochlear ablation. J Neurophysiol 53:1483-1500.

Klumpp RG, Eady HR (1956) Some measurements of interaural time difference thresholds. J Acoust Soc Am 28:859-860.

Kuwada S, Yin TC (1983) Binaural interaction in low-frequency neurons in inferior colliculus of the cat. I. Effects of long interaural delays, intensity, and repetition rate on interaural delay function. J Neurophysiol 50:981-999.

Kuwada S, Yin TC, Wickesberg RE (1979) Response of cat inferior colliculus neurons to binaural beat stimuli: possible mechanisms for sound localization. Science 206:586-588.

Lawson DT, Wilson BS, Zerbi M, van den Honert C, Finley CC, Farmer Jr JC, McElveen Jr JT, Roush PA (1998) Bilateral cochlear implants controlled by a single speech processor. Am J Otol 19:758-761.

Le Beau FE, Rees A, Malmierca MS (1996) Contribution of GABA- and glycine-mediated inhibition to the monaural temporal response properties of neurons in the inferior colliculus. J Neurophysiol 75:902-919.

Litovsky R, Parkinson A, Arcaroli J, Sammeth C (2006) Simultaneous bilateral cochlear implantation in adults: a multicenter clinical study. Ear Hear 27:714-731.

Litvak LM, Smith ZM, Delgutte B, Eddington DK (2003) Desynchroniza- 
tion of electrically evoked auditory-nerve activity by high-frequency pulse trains of long duration. J Acoust Soc Am 114:2066-2078.

Long CJ, Eddington DK, Colburn HS, Rabinowitz WM (2003) Binaural sensitivity as a function of interaural electrode position with a bilateral cochlear implant user. J Acoust Soc Am 114:1565-1574.

Macpherson EA, Middlebrooks JC (2002) Listener weighting of cues for lateral angle: the duplex theory of sound localization revisited. J Acoust Soc Am 111:2219-2236.

McAlpine D, Jiang D, Shackleton TM, Palmer AR (2000) Responses of neurons in the inferior colliculus to dynamic interaural phase cues: evidence for a mechanism of binaural adaptation. J Neurophysiol 83:1356-1365.

McAlpine D, Jiang D, Palmer AR (2001) A neural code for low-frequency sound localization in mammals. Nat Neurosci 4:396-401.

Merzenich MM, Reid MD (1974) Representation of the cochlea within the inferior colliculus of the cat. Brain Res 77:397-415.

Moxon EC (1967) Electric stimulation of the cat's cochlea: a study of discharge rates in single auditory nerve fibers. In: Electrical engineering. Cambridge, MA: MIT.

Muller J, Schon F, Helms J (2002) Speech understanding in quiet and noise in bilateral users of the MED-EL COMBI 40/40+ cochlear implant system. Ear Hear 23:198-206.

Nuding SC, Chen GD, Sinex DG (1999) Monaural response properties of single neurons in the chinchilla inferior colliculus. Hear Res 131:89-106.

Oliver DL, Beckius GE, Bishop DC, Loftus WC, Batra R (2003) Topography of interaural temporal disparity coding in projections of medial superior olive to inferior colliculus. J Neurosci 23:7438-7449.

Parkins CW (1989) Temporal response patterns of auditory nerve fibers to electrical stimulation in deafened squirrel monkeys. Hear Res 41:137-168.

Poon BB (2006) Sound localization and interaural time sensitivity with bilateral cochlear implants. In: Harvard-MIT Division of Health Sciences and Technology. Cambridge, MA: MIT.

Sachs MB, Abbas PJ (1974) Rate versus level functions for auditory-nerve fibers in cats: tone-burst stimuli. J Acoust Soc Am 56:1835-1847.

Sakitt B (1973) Indices of discriminability. Nature 241:133-134.

Schon F, Muller J, Helms J (2002) Speech reception thresholds obtained in a symmetrical four-loudspeaker arrangement from bilateral users of MED-EL cochlear implants. Otol Neurotol 23:710-714.

Schroeder MR (1977) New viewpoints in binaural interactions. In: Psychophysics and physiology of hearing (Evans EF, Wilson JP, eds), pp 455467. New York: Academic.

Semple MN, Kitzes LM (1987) Binaural processing of sound pressure level in the inferior colliculus. J Neurophysiol 57:1130-1147.

Shackleton TM, Skottun BC, Arnott RH, Palmer AR (2003) Interaural time difference discrimination thresholds for single neurons in the inferior colliculus of guinea pigs. J Neurosci 23:716-724.

Shamma SA, Shen NM, Gopalaswamy P (1989) Stereausis: binaural processing without neural delays. J Acoust Soc Am 86:989-1006.

Shepherd RK, Baxi JH, Hardie NA (1999) Response of inferior colliculus neurons to electrical stimulation of the auditory nerve in neonatally deafened cats. J Neurophysiol 82:1363-1380.

Smith PH, Joris PX, Carney LH, Yin TC (1991) Projections of physiologically characterized globular bushy cell axons from the cochlear nucleus of the cat. J Comp Neurol 304:387-407.

Smith ZM, Delgutte B (2003a) Binaural interactions with bilateral electric stimulation of the cochlea: evoked potential and single-unit measures. Paper presented at Association for Research in Otolaryngology Midwinter Meeting, Daytona Beach, FL, February.

Smith ZM, Delgutte B (2003b) Neural sensitivity to interaural timing differences with bilateral electric stimulation of the cochlea. Paper presented at Conference on Implantable Auditory Prosthesis, Pacific Grove, CA, August.

Smith ZM, Delgutte B (2005a) Binaural interactions in the auditory mid- brain with bilateral cochlear implants. Paper presented at Association for Research in Otolaryngology Midwinter Meeting, New Orleans, LA, February.

Smith ZM, Delgutte B (2005b) What to do with the "where": a phsyiologically inspired strategy for delivering interaural timing cues with bilateral cochlear implants. Paper presented at Conference on Implantable Auditory Prosthesis, Pacific Grove, CA, July.

Snyder R, Leake P, Rebscher S, Beitel R (1995) Temporal resolution of neurons in cat inferior colliculus to intracochlear electrical stimulation: effects of neonatal deafening and chronic stimulation. J Neurophysiol 73:449-467.

Snyder RL, Rebscher SJ, Cao KL, Leake PA, Kelly K (1990) Chronic intracochlear electrical stimulation in the neonatally deafened cat. I: Expansion of central representation. Hear Res 50:7-33.

Snyder RL, Rebscher SJ, Leake PA, Kelly K, Cao K (1991) Chronic intracochlear electrical stimulation in the neonatally deafened cat. II. Temporal properties of neurons in the inferior colliculus. Hear Res 56:246-264.

Spitzer MW, Semple MN (1991) Interaural phase coding in auditory midbrain: influence of dynamic stimulus features. Science 254:721-724.

Spitzer MW, Semple MN (1998) Transformation of binaural response properties in the ascending auditory pathway: influence of time-varying interaural phase disparity. J Neurophysiol 80:3062-3076.

Tollin DJ, Yin TC (2005) Interaural phase and level difference sensitivity in low-frequency neurons in the lateral superior olive. J Neurosci 25:10648-10657.

Vale C, Sanes DH (2002) The effect of bilateral deafness on excitatory and inhibitory synaptic strength in the inferior colliculus. Eur J Neurosci 16:2394-2404.

van den Honert C, Stypulkowski PH (1987) Temporal response patterns of single auditory nerve fibers elicited by periodic electrical stimuli. Hear Res 29:207-222.

van Hoesel R, Ramsden R, Odriscoll M (2002) Sound-direction identification, interaural time delay discrimination, and speech intelligibility advantages in noise for a bilateral cochlear implant user. Ear Hear 23:137-149.

van Hoesel RJ (2007) Sensitivity to binaural timing in bilateral cochlear implant users. J Acoust Soc Am 121:2192-2206.

van Hoesel RJ, Tyler RS (2003) Speech perception, localization, and lateralization with bilateral cochlear implants. J Acoust Soc Am 113:1617-1630.

Widrow B, Glover JR, Mccool JM, Kaunitz J, Williams CS, Hearn RH, Zeidler JR, Dong E, Goodlin RC (1975) Adaptive noise canceling-principles and applications. Proc IEEE 63:1692-1716.

Wightman FL, Kistler DJ (1992) The dominant role of low-frequency interaural time differences in sound localization. J Acoust Soc Am 91:1648-1661.

Wilson BS, Lawson DT, Muller JM, Tyler RS, Kiefer J (2003) Cochlear implants: some likely next steps. Annu Rev Biomed Eng 5:207-249.

Xu SA, Shepherd RK, Chen Y, Clark GM (1993) Profound hearing loss in the cat following the single co-administration of kanamycin and ethacrynic acid. Hear Res 70:205-215.

Yin TC, Chan JC (1990) Interaural time sensitivity in medial superior olive of cat. J Neurophysiol 64:465-488.

Yin TC, Kuwada S (1983) Binaural interaction in low-frequency neurons in inferior colliculus of the cat. II. Effects of changing rate and direction of interaural phase. J Neurophysiol 50:1000-1019.

Yin TC, Chan JC, Irvine DR (1986) Effects of interaural time delays of noise stimuli on low-frequency cells in the cat's inferior colliculus. I. Responses to wideband noise. J Neurophysiol 55:280-300.

Yost WA, Wightman FL, Green DM (1971) Lateralization of filtered clicks. J Acoust Soc Am 50:1526-1531.

Zurek PM (1993) Binaural advantages and directional effects in speech intelligibility. In: Acoustical factors affecting hearing aid performance (Studebaker GA, Hochberg I, eds), pp 255-276. Boston: Allyn and Bacon. 